\title{
Osteoarthritic Infrapatellar Fat Pad Aggravates Cartilage Degradation via Activation of p38MAPK and ERK1/2 Pathways
}

\section{Zuoqing Zhou}

Southern Medical University

\section{Su'an Tang}

Southern Medical University

Xiaoyu Nie

Southern Medical University

Yiqun Zhang

Shaoyang University

Delong Li

Southern Medical University

Yang Zhao

Southern Medical University

Yumei Cao

Southern Medical University

Jianwen Yin

Shaoyang University

Tianyu Chen

Southern Medical University

Guangfeng Ruan

Southern Medical University

Zhaohua Zhu

Southern Medical University

Xiaochun Bai

Southern Medical University

\section{Weiyu Han}

Southern Medical University

Changhai Ding ( $\nabla$ changhai.ding@utas.edu.au )

University of Tasmania Menzies Institute for Medical Research https://orcid.org/0000-0002-9479-730X 
Keywords: Osteoarthritis, Infrapatellar fat pad, Cytokines, p38MAPK, ERK1/2

Posted Date: November 2nd, 2020

DOI: https://doi.org/10.21203/rs.3.rs-98317/v1

License: (c) (i) This work is licensed under a Creative Commons Attribution 4.0 International License. Read Full License 


\section{Abstract}

Background: Although existing studies have suggested the involvement of the infrapatellar fat pad (IPFP) during the development of knee osteoarthritis (OA), the role of IPFP is still controversial. This study aimed to investigate the biochemical effects of osteoarthritic IPFP on cartilage and the underlying mechanisms.

Methods: Human IPFP and articular cartilage were collected from end-stage OA patients during total knee arthroplasty. IPFP derived fat-conditioned medium (FCM) was used to stimulate human primary chondrocytes and cartilage explants. CCK8 was used to detect the viability of human chondrocyte. qRTPCR and western blotting was performed to evaluate the balance of extracellular matrix (ECM) catabolism and anabolism in human chondrocytes with FCM stimulation. Functional effect of osteoarthritic IPFP was also demonstrated in human articular cartilage by ex vivo assay. Activation of relative pathways and its effects on chondrocytes were assessed through immunoblotting and inhibition experiments, respectively. Neutralization test was performed to identify the main factors and their associated pathways responsible for the effects of IPFP.

Results: Osteoarthritic IPFP-derived FCM significantly induced extracellular matrix (ECM) degradation in both human primary chondrocytes and cartilage explants. Several pathways, such as NF-KB, mTORC1, p38MAPK, JNK, and ERK1/2 signaling were significantly activated in human chondrocytes with osteoarthritic IPFP-derived FCM stimulation. Interestingly, inhibition of p38MAPK and ERK1/2 signaling pathway could alleviate the detrimental effects of FCM on chondrocytes while inhibition of other signaling pathways had no similar results. In addition, IL-1 $\beta$ and TNF- $\alpha$ instead of IL- 6 in osteoarthritic IPFP-derived FCM played a key role in cartilage degradation via activating p38MAPK rather than ERK1/2 signaling pathway.

Conclusions: Osteoarthritic IPFP induces the degradation and inflammation of cartilage via activation of p38MAPK and ERK1/2 pathways, in which IL-1 $\beta$ and TNF- $\alpha$ act as the key factors. Our study suggests that modulating the effects of IPFP on cartilage may be a promising strategy for knee OA intervention.

\section{Introduction}

Osteoarthritis $(\mathrm{OA})$ is the most frequent joint disorder, characterized by structural alterations involving the whole joint. The pathogenesis of OA includes cartilage degradation, subchondral bone remodeling, osteophyte formation and synovial inflammation, which leads to joint pain, tenderness, swelling, stiffness and limitation of movement [1, 2]. It has caused a huge socioeconomic burden worldwide due to its disabling characteristics and an unmet medical need $[3,4]$. Investigation into the pathogenesis of $O A$ and searching for the novel prevention and treatment strategies are of great significance.

Infrapatellar fat pad (IPFP) is the largest local adipose tissue around the knee joint. Researchers found that IPFP released a variety of bioactive factors including pro-inflammatory and anti-inflammatory mediators as well as catabolic and anabolic components. It acts as an important source of cytokines in knee synovial fluids $[5,6]$. Our previous studies revealed that IPFP maximal area as well as a larger 
volume measured by magnetic resonance imaging had a protective role for the knee symptoms and cartilage degradation [13-16], while signal intensity alterations within IPFP facilitated the progression of OA [17-20]. However, there are still controversies about the role of IPFP in OA and further studies are warranted.

It has been reported that identified risk factors including aging, joint injury, genetics and obesity initiate or promote $\mathrm{OA}$ by activating many different molecular pathways [21]. IPFP-derived factors contain IL-1 $\beta$, TNF-a, IL-6, growth factors, adipocytokines and other known or unknown components, which may also activate different pathways to interact with cartilage or other adjacent tissues in advanced OA [22]. Some of them are well investigated; however, the role of IPFP released factors in the progression of OA is poorly clarified and the underlying biochemical mechanisms remain unclear.

In this study, we aimed to investigate the biochemical effects of osteoarthritic IPFP on cartilage by measuring its effects on the expression of cartilage degrading enzymes and inflammatory mediators, and to identify the leading cytokines as well as the potential signal transduction pathways.

\section{Methods}

\section{Articular cartilage and IPFP collection}

This study was approved by the Ethics Committee of Zhujiang Hospital of Southern Medical University (Guangzhou, China). Human IPFP and cartilage were aseptically collected as surgical waste from endstage knee OA patients $(n=23)$ who had undergone total knee arthroplasty. Informed consent form was signed by each patient before the surgery. Further information about the individuals was summarized in the Supplementary Table 1.

\section{Preparation of infrapatellar fat pad-conditioned medium (FCM)}

To generate FCM, the inner part of the IPFP was carefully separated from the synovium lining. Then the fat pad, minced into pieces of approximately $50 \mathrm{mg}$, was immediately incubated in Dulbecco's Modified Eagle's Medium/F12 (DMEM/F12) containing $0.75 \mathrm{mg} / \mathrm{ml} \mathrm{BSA}, 100 \mathrm{U} / \mathrm{ml}$ penicillin, and $0.1 \mathrm{mg} / \mathrm{ml}$ streptomycin at a concentration of $100 \mathrm{mg} / \mathrm{ml}$. After incubation for $2 \mathrm{~h}$, the medium was refreshed to remove possible contamination. In another $24 \mathrm{~h}$, the supernatants were harvested, centrifuged at $300 \mathrm{~g}$ for 8 min and frozen at $-80^{\circ} \mathrm{C}$ until use.

\section{Isolation and culture of chondrocytes}

To isolate the human articular chondrocytes, knee cartilage was washed with phosphate-buffered saline and cut into small particles with a diameter about $1 \mathrm{~mm}$. Subsequently, the cartilage tissues were 
digested in trypsin-EDTA solution containing $0.25 \%$ trypsin and $0.02 \%$ EDTA for 30 min at $37{ }^{\circ} \mathrm{C}$ with gentle agitation, followed by a digestion in $0.2 \%$ type 2 collagenase overnight. The digested suspension was then filtered through a 40- $\mu \mathrm{m}$ mesh and centrifuged at $300 \mathrm{~g}$ for $5 \mathrm{~min}$. Afterwards, cell pellets were resuspended and seeded in $25 \mathrm{~cm}^{2}$ culture flasks. The medium was refreshed every 2 days.

\section{RNA extraction and quantitative real-time polymerase chain reaction (qRT-PCR)}

Total RNA was isolated from treated chondrocytes using an RNAiso Plus kit (Takara), and then was reverse transcribed into cDNA using a PrimeScript ${ }^{\mathrm{TM}}$ RT Master Mix kit (Takara). Afterwards, cDNA was amplified by qRT-PCR using TB Green ${ }^{\mathrm{TM}}$ Premix Ex Taq $^{T M}$ (Takara) and LightCycler 480 System (Roche) with specific primers. The glyceraldehyde 3-phosphate dehydrogenase (GAPDH) was used as an internal control gene. Results were calculated by $2^{-} \triangle \triangle \mathrm{Ct}$ formula and presented as fold changes relative to GAPDH. Primer sequences are listed in Supplementary Table 2.

\section{Immunoblotting}

Chondrocytes were lysed with RIPA lysis buffer (KeyGEN) supplemented with protease inhibitors. Total protein was collected and denatured. After a $10 \%$ sodium dodecyl sulfate-polyacrylamide gel electrophoresis, proteins were transferred to a polyvinylidene difluoride membrane (Millipore). Blots were further probed with primary antibodies (listed in Supplementary Table 3) and horseradish peroxidaseconjugated secondary antibodies (ABclonal). After washing, the membrane was visualized using an enhanced chemiluminescence kit ( $\mathrm{Ncm}$ Biotech).

\section{Histological and immunohistochemical analysis}

Articular cartilage was diced into small pieces which were randomly and evenly divided into two groups, one was treated with autologous FCM, the other with the medium that was used to generate the FCM. The medium was changed every 2 days. After 14 days of culture, the cartilage explants were fixed and decalcified. Subsequently, the tissues were embedded in paraffin and sectioned at 4- $\mu$ m thickness. The histological sections were stained to detect the histomorphology and extracellular matrix of cartilage explants with Safranin O/Fast Green (Sigma) as well as Toluidine blue (Solarbio). For immunohistochemical $(\mathrm{IHC})$ analysis, the sections were stained overnight at $4{ }^{\circ} \mathrm{C}$ with the following primary antibodies: rabbit anti-MMP3 (1:50, Abcam, ab52915), rabbit anti-COX2 (1:200, Abcam, ab179800), and rabbit anti-Collagen II (1:200, Abcam, ab34712). More detailed information of the procedure is provided in supplementary materials.

\section{Inhibitor experiments}


The effect of relative pathway activation on chondrocytes was examined through pathway inhibition. To assess the pathway-inhibited effects of different inhibitors at appropriate concentrations, cells were pretreated for $2 \mathrm{~h}$ with the following pathway inhibitors: BAY 117082 (BAY, $1 \mu \mathrm{M}$, an NF-kB inhibitor, Selleck), Rapamycin (Rapa, 10 nM, an mTORC1 inhibitor, Sigma), SB203580 (SB, $10 \mu M$, a p38MAPK inhibitor, Selleck), SP 600125 (SP, $5 \mu \mathrm{M}$, a JNK inhibitor, MCE), and U0126 (U0, $10 \mu \mathrm{M}$, an ERK inhibitor, $M C E)$, respectively. Then, these chondrocytes were further stimulated with or without $50 \%$ FCM for 30 min in the presence of relative pathway inhibitors. Proteins were collected to do the next western blotting analysis.

To detect the influence of relative pathway inhibition on expressions of degrading enzymes and proinflammatory cytokines in chondrocytes, cells were treated with or without autologous 50\% FCM for $24 \mathrm{~h}$ following pretreatment with relative pathway inhibitor for $2 \mathrm{~h}$. And then, total RNA and proteins were extracted to do the further qRT-PCR or western blotting analysis.

\section{Neutralization tests}

An early study suggested that adipokines such as leptin and adiponectin secreted by IPFP may not be responsible for cartilage destruction and inflammation [23]. To identify the main factors that mediated the FCM-induced degradation and inflammation of cartilage, neutralization experiments were performed. Osteoarthritic IPFP could secret higher levels of inflammatory mediators such as IL- 6 and TNF- $a$ compared with paired subcutaneous adipose tissues, and IL-1 $\beta$, IL- 6 and TNF-a have long been viewed as the main inflammatory factors in OA pathophysiology [7]. To this end, we first chose neutralizing antibodies targeting these cytokines.

FCMs were incubated overnight with neutralizing antibodies targeting human IL-1 $\beta$, IL-6, and TNF-a, respectively. FCM incubated with normal IgG was set as the negative control. Subsequently, chondrocytes were stimulated with relative FCM for $24 \mathrm{~h}$, and then the total RNA and proteins were extracted to further analyze the expressions of degrading enzymes and inflammatory mediators. The information of neutralizing antibodies is depicted in Supplementary Table 3.

\section{Statistical analysis}

All experiments were conducted in triplicate. Differences between two groups were analyzed through Student's $t$-test while those among three or more groups were analyzed by one-way analysis of variance (ANOVA). All statistical analyses were performed with SPSS 24.0. Data were presented as the mean \pm SD, and $P<0.05$ was considered as significant.

\section{Results}




\section{Osteoarthritic IPFP-derived FCM induces catabolic and inflammatory phenotypes in human primary chondrocytes}

The role of IPFP-derived factors on chondrocytes remains controversial. Therefore, we determined the effect of FCM to human articular chondrocytes firstly. FCM that was harvested by incubation of IPFP pieces, was subsequently added to human primary chondrocytes at different concentrations (Fig. 1A). Little effect of IPFP-derived FCM on cell viability was observed by CCK8 assay (Supplementary Figure S1). qRT-PCR indicated that FCM upregulated the expression of ECM catabolic markers including MMP1, $M M P 3$, and ADAMTS4 in a dose-dependent manner (Fig. 1B). Similarly, the expression of inflammatory genes such as $I L-1 B, I L-6$ and COX2 were increased consistently, especially in the $100 \%$ FCM group (Fig. 1C). Moreover, we examined the expression of chondrogenic-specific genes (such as SOX9, COL2A1, and $A C A M$ ). Only SOX9 and COL2A1 showed a trend of downregulation in the concentration $100 \%$ and little effects were detected on the expression of $\operatorname{ACAN}$ (Fig. 1D).

In consistent with qRT-PCR results, the protein levels of MMP1, MMP3, ADAMTS4, and COX2 in chondrocytes were significantly enhanced with the increase of FCM concentrations (Fig. 1E-I). Taken together, our data indicate that osteoarthritic IPFP-derived FCM could induce a catabolic and inflammatory phenotype in human chondrocytes.

\section{FCM promotes the degradation of cartilage matrix and aggravates the inflammation of cartilage ex vivo}

To investigate the effects of osteoarthritic IPFP on cartilage tissue, cartilage explants were treated with or without IPFP-derived FCM ex vivo for 14 days (Fig. 2A). Loss of proteoglycans was detected through safranin o/fast green-staining in FCM-treated cartilage explants but no significant cartilage erosion compared with control group (non FCM-treated group) (Fig. 2B). The similar result can be determined by toluidine blue staining of the cartilage slices (Fig. 2C). Subsequently, we performed IHC staining and observed a decrease in Collagen II in FCM-treated tissues (Fig. 2D). Furthermore, we also examined markers of inflammation and degradation through $\mathrm{IHC}$ in cartilage explants. The number of MMP3 or COX2 positive cells increased significantly in the samples treated with FCM compared with the control (Fig. 2E-H). Taken together, our data suggest that osteoarthritic IPFP plays a detrimental role in cartilage degradation.

\section{Signaling pathways associated with knee OA progression were activated within FCM stimulated chondrocytes}

To explore the mechanisms behind the detrimental effects of FCM derived from advanced knee OA on cartilage, some classic signaling pathways being reported to be associated with OA were assessed [2426]. P65, a member in NF-kB family which plays a pivotal role in inflammatory and immune responses, 
was significantly phosphorylated within 5-60 min after stimulation with FCM (Fig. 3A). S6, a downstream molecule of mTOC1, was also phosphorylated in the presence of FCM (Fig. 3B). At the same time, phosphorylation levels of markers in all three pathways of MAPK including P38, JNK, and ERK1/2 were enhanced by FCM at different time points (Fig. 3C-E). These results obtained by western blotting analysis suggest that the biochemical effects of FCM on cartilage are probably mediated by one or more pathways above.

\section{p38MAPK and ERK1/2 pathways are responsible for osteoarthritic IPFP induced cartilage degradation}

To study which signaling pathway plays a key role in the damage of IPFP-derived FCM, signaling inhibitors were applied. First, we successfully identified pathway-inhibitory effects of these inhibitors at appropriate concentrations through western blotting (Fig. 4A-E). FCM-mediated activations of signaling pathways including NF-KB, mTORC1, p38MAPK, JNK, and ERK1/2 were significantly inhibited by BAY ( $1 \mu \mathrm{M}$, an NF-KB inhibitor), Rapa (10 nM, an mTORC1 inhibitor), SB (10 $\mu \mathrm{M}$, a p38MAPK inhibitor), SP ( $5 \mu \mathrm{M}$, a JNK inhibitor), and UO (10 $\mu \mathrm{M}$, an ERK inhibitor), respectively. Of note, SB suppressed the phosphorylation of MAPKAPK2, a downstream protein of p38MAPK, instead of p38.

Subsequently, human chondrocytes were cultured for $24 \mathrm{~h}$ with IPFP-derived FCM after pretreatment with or without inhibitors for $2 \mathrm{~h}$. Total RNA and proteins were extracted to determine the expression of ECM catabolic and inflammatory markers. The mRNA expression of ECM degrading enzymes including MMP1, $M M P 3$, and ADAMTS4 were substantially upregulated in chondrocytes co-cultured with FCM, while downregulated in the presence of SB or U0 except for ADAMTS4 (Fig. 4F). Inflammatory mediators including $I L-1 B, I L-6$ and $C O X 2$ were also upregulated after stimulation by FCM. In the groups pretreated with SB or U0, the expression of $I L-6$ and COX2 was decreased, while $I L-1 B$ expression was downregulated only in the presence of UO (Fig. 4G). These results were further supported by protein expressions including MMP1, MMP3, and COX2 (Fig. 4H). Taken together, these findings suggest that p38MAPK and ERK1/2 pathways rather than NF-KB, mTORC1 or JNK are involved in the adverse effects of FCM on articular chondrocytes.

\section{Pro-inflammatory and pro-catabolic effects of FCM on articular chondrocytes are reduced after neutralization of IL- $1 \beta$ and TNF- $a$, not IL- 6}

To identify the main factors that mediate the inflammatory and degradative effect of FCM, human chondrocytes were cultured with FCM which was pretreated with or without a neutralizing antibody or a control IgG. Consistently, osteoarthritic IPFP-derived FCM enhanced the expression of catabolic and inflammatory genes, which was almost unaffected by normal mouse IgG (Fig. 5A, B). As expected, neutralizing IL-1 $\beta$ or TNF- $\alpha$ in FCM resulted in the decrease of ECM degrading enzymes and inflammatory 
markers, though only a trend for MMP1 mRNA after blocking IL-1 $\beta$ was observed. The decrease became even more pronounced when the multiple antibodies were used (Fig. 5A, B). Surprisingly, the addition of IL-6 neutralizing antibody to FCM before stimulation of chondrocytes had little impact on the effects of FCM (Fig. 5A, B). Similarly, these results were further supported by western blotting analysis (Fig. 5C). Our data suggest that IL-1 $\beta$ and TNF-a, instead of IL-6, might play a decisive role in the pro-catabolic and proinflammatory effects of FCM.

Next, to test whether these factors regulated the effects of FCM via the identified pathways, neutralization experiments towards IL-1 $\beta$, TNF- $\alpha$, IL- 6 or all of them were conducted. Blocking IL-1 $\beta$ or TNF- $\alpha$ in FCM could inhibit the phosphorylation of P38 and P65, with the exception of ERK1/2, suggesting both IL-1 $\beta$ and TNF-a were involved in the activation of p38MAPK and NF-KB (Fig. 5D, F). However, of these two pathways, only p38MAPK was the identified pathway that mediate the effects of IPFP (in combination with the data shown above). Therefore, we speculated that both IL-1 $\beta$ and TNF-a functioned via activating p38MAPK signaling pathway. In contrast, the phosphorylation of P65, P38 and ERK1/2 remained unchanged upon the neutralization of IL-6, suggesting IL-6 might not be critical (Fig. 5E). Actually, simultaneous blockade of IL- $1 \beta$, TNF- $\alpha$ and IL- 6 can also lead to inhibition of the p38MAPK and NF-KB signaling, but not the ERK1/2 (Fig. 5G), which was in agreement with above results. Overall, both IL-1 $\beta$ and TNF- $a$ functioned via activating p38MAPK while IL- 6 might not be the main factor in ECM catabolism and inflammation induced by FCM.

\section{Discussion}

Although emerging evidences suggest that IPFP may involve in the onset and progression of knee OA [27], existing studies focusing on the cross-talk between IPFP and adjacent tissues such as cartilage are controversial, and the underlying mechanisms have not been elucidated, leading to a confusion whether to remove the IPFP during total knee arthroplasty. In the current study, we explored the biochemical effects of human osteoarthritic IPFP on cartilage by examining the responses of chondrocytes and cartilage explants to IPFP-derived FCM. Our data show that IPFP, at the end-stage of knee OA, has a proinflammatory and pro-catabolic phenotype for adjacent cartilage. The possible mechanism is that IPFP released factors activate different molecular pathways, especially the p38MAPK and ERK $1 / 2$ signaling, among which, IL-1 $\beta$ and TNF- $\alpha$ are the key drivers (Fig. 6).

After stimulating chondrocytes or cartilage with FCM derived from autologous IPFP, the expressions of chondro-degrading enzymes as well as inflammatory mediators were significantly upregulated, while the chondrocyte viability and chondrogenic-specific genes were weakly affected. Of note, our study demonstrated that there is no significant change in chondrocyte viability with IPFP treatment. In contrast with our data, a previous study found that IPFP adipocytes released factors could induce a dosedependent decrease in chondrocyte number and downregulate the mRNA level of chondrogenic-specific genes [28]. In addition to adipocytes, other cell types such as fibroblasts, macrophages and leucocytes in IPFP may also contribute to IPFP's effects, which could explain the discrepancy. Similarly, the same study also indicated that co-culturing with IPFP adipocytes, chondrocytes showed an enhanced expression of 
genes including $I L-1 B, C O X 2, M M P 1$ and $M M P 3$, suggesting that the inflammatory and matrix degrading effects of IPFP may largely depend on the adipocytes [28]. Conversely, Bastiaansen-Jenniskens et al. reported that IPFP from patients with end-stage OA inhibited catabolic metabolism of bovine cartilage [10]. Interestingly, others did the similar experiments and came to the opposite conclusion [29]. However, in these two studies, healthy bovine cartilage instead of paired human OA cartilage was used, which might not really imitate a pathologic changes of OA. Furthermore, a cross-use of samples from different species should not be ignored. Species-specific regulations have been reported by other studies [30], especially, for bovine and human chondrocyte [31]. Actually, during the interaction between cartilage and normal IPFP, differences in the condition of cartilage may lead to completely different results, which was identified in bovine $[11,12]$. Our data are in agreement with those presented by Gross et al. who found detrimental effects of IPFP-derived FCM on human chondrocyte in spite of the heterogeneity that might happen in human samples from different individuals [23]. Here, we examined the role of osteoarthritic IPFP not only through cellular tests but also experiments ex vivo, reaching the consistent conclusion. Moreover, the underlying mechanisms were explored. It is well established that the role of IPFP during OA includes the aspects of biomechanics, biochemistry, and damage repair [32, 33]. However, this study mainly focused on its biochemical effects at the end-stage of knee OA. Further researches are warranted to assess the combined role of biomechanics and biochemistry within IPFP.

To the best of our knowledge, this is the first study to investigate the signaling pathways that mediate the interaction between osteoarthritic IPFP and chondrocytes. Our data indicate that p38MAPK and ERK1/2 might be the main pathways responsible for the pro-inflammatory and pro-catabolic effects of osteoarthritic IPFP, though all five examined pathways including NF-KB, mTORC1, p38MAPK, JNK and ERK1/2 are activated by FCM in chondrocyte. NF-KB signaling has long been viewed as the essential pathway, which is involved in cartilage inflammation and matrix degradation during the process of $O A$ [24]. Chen et al. reported that medium conditioned by IPFP adipocytes could induce the activation of NF$\mathrm{KB}$ signaling, increasing the level of inflammatory markers in chondrocyte[28]. However, further reverse effect by blocking the pathway has not been verified in their study. In our data, NF-KB pathway seems not to be the main player that mediates the effects of IPFP, because NF-KB inhibition could not improve the inflammatory and degradative effects of FCM. This may also be attribute to the complexity of the components contained in FCM and the counteraction from other pathways. An earlier study has demonstrated that leptin combined with IL-1 activates MAPK and NF-KB signaling in chondrocytes [29]. This study supports our results because both leptin and IL-1 are contained in FCM. mTORC1 pathway also plays an key role in cartilage metabolism and pathophysiology of OA [25]. The activation of mTORC1 in chondrocyte by gene modification could initiate OA in mice [34]. However, in the current study, we did not find any improvement in the inflammatory and chondro-destructive markers after inhibiting mTORC1 in FCM-induced chondrocytes, suggesting mTORC1 might not be the main signaling that mediate IPFP-induced effects.

MAPK is a key upstream signaling leading to cartilage destruction, including members of p38MAPK, JNK and ERK1/2 [35]. Sondergaard et al. reported both p38MAPK and ERK1/2 signaling were essential for MMPs expression and activity, while only ERK1/2 was essential for aggrecanase-mediated degradation 
[26]. This could explain why the expression of MMP1 and MMP3 was significantly downregulated when p38MAPK or ERK1/2 was inhibited in our study. Moreover, our results showed ERK1/2 rather than p38MAPK inhibition could downregulate ADAMTS4 expression though only a trend was observed, which is also in accordance with the finding by Sondergaard et al [26]. Although JNK signaling may function in the process of OA [36], an early study found that JNK pathway might be of little or no importance for IL$1 \beta$-induced IL-6 expression [37]. Hui et al. suggested JNK inhibition had no effect on MMP13 expression of chondrocyte stimulated by leptin, as well as cartilage collagen release induced by leptin and IL-1 [29]. Similarly, in our study, JNK inhibition showed no effect on the improvement of cartilage damage markers induced by FCM. Overall, these data highlighted that the role of JNK might not be as important as that of p38MAPK or ERK1/2 in FCM-induced cartilage damage. Of the whole five pathways examined, p38MAPK and ERK1/2 rather than mTORC1, NF-KB and JNK are the main pathways that mediate the biochemical effects of IPFP on cartilage. However, further researches are required to provide more information on the signaling mechanisms underlying the interaction between IPFP and cartilage.

IPFP-derived FCM is considered as a black box due to its diverse components [10]. An early publication showed that the leptin in IPFP-derived FCM might be able to induce the expression of MMPs in chondrocytes [29]; however, this may be limited by a small sample size and the bias in case selection. More recently, researchers demonstrated the effect of IPFP on chondrocyte was not mediated by leptin or adiponectin [23]. Therefore, we speculate that the deleterious phenotype of osteoarthritic IPFP mainly mediated by other cytokines instead of adipocytokines. Because IL-1 $\beta$, TNF- $\alpha$ and IL- 6 are widely considered as the main pro-inflammatory cytokines during OA progression and could be highly secreted by IPFP [5, 22], we performed neutralization test to determine whether these factors are the leading players contributing to the biochemical deleterious effects of IPFP on cartilage. As a result, neutralizing IL-1 $\beta$ or TNF-a could attenuate the pro-inflammatory and pro-catabolic effects of FCM on chondrocytes. Surprisingly, blocking IL- 6 did not change these adverse effects, suggesting that both IL-1 $\beta$ and TNF- $a$ rather than IL-6 might play decisive roles in IPFP-induced damage. Therefore, we deduce that IL- 6 may play a subordinate role in IPFP-derived FCM and other protective factors such as IL-10, adiponectin and oxylipins secreted by IPFP may also counteract its effects. In addition, the signaling pathways especially p38MAPK and ERK1/2 responsible for the adverse effects of FCM showed no alteration after neutralizing IL-6 in FCM, which further suggested that IL-6 might not be a key player in the pro-inflammatory and procatabolic phenotype of IPFP. In our study, we did not detect all the factors that may mediate the damage effect of IPFP; however, what we need most is to identify the leading factors in IPFP, which is of the utmost importance to guide the prevention and treatment of knee $\mathrm{OA}$.

\section{Conclusion}

In conclusion, osteoarthritic IPFP enhances the inflammation and degradation of cartilage through activating relative signaling pathways, especially p38MAPK and ERK1/2. Cytokines including IL-1 $\beta$ and TNF-a secreted by IPFP play key roles in cartilage damage. These findings suggest that modulating the effects of IPFP on cartilage may be a promising strategy for knee OA intervention. 


\section{Abbreviations}

ACAN: Aggrecan; ADAMTS: A disintegrin and metalloproteinase with thrombospondin-like motifs; CCK8: Cell counting kit-8; cDNA: Complementary DNA; COL2A1: Type II collagen alpha 1 chain; COX2: Cyclooxygenase 2; DMEM: Dulbecco's modified Eagle's medium; EDTA: Ethylene diamine tetraacetic acid; ECM: Extracellular matrix; ERK: Extracellular signal-regulated kinase; FCM: Fat-conditioned medium; GAPDH: Glyceraldehyde 3-phosphate dehydrogenase; IL: Interleukin; IPFP: Infrapatellar fat pad; JNK: CJun-N-terminal kinase; MAPK: Mitogen-activated protein kinase; MMP: Matrix metalloproteinase; mTORC1: Mammalian target of rapamycin complex 1; MAPKAPK2: Recombinant mitogen activated protein kinase activated protein kinase 2. NF-KB: Nuclear factor kappa B; OA: Osteoarthritis; qRT-PCR: Quantitative real-time polymerase chain reaction; SD: Standard deviation; TNF-a: Tumor necrosis factor a.

\section{Declarations}

\section{Ethics approval and consent to participate}

The study was approved by the regional ethics committee of Zhujiang Hospital of Southern Medical University (2019KY02203). All patients signed written informed consent.

\section{Consent for publication}

Not applicable

\section{Availability of data and materials}

The datasets used during the current study are available from the corresponding author on reasonable request.

\section{Competing interest}

The authors declare no conflict of interest.

\section{Funding}

This study was financially supported by grants from the National Natural Science Foundation of China (81773532, 81974342).

\section{Authors' contributions}


ZZ: conception and design, collection of data, manuscript writing; ST: conception and design, data analysis and manuscript writing; $X N, Y Z, D L$ : collection of data, data analysis; $Y Z, Y C, J Y, G R$ : collection of data; ZZ, TC, XB, WH: provision of study material. CD: conception and design, obtaining the grant, final approval of manuscript. All authors critically appraised the manuscript and agreed to the final submission.

\section{Acknowledgments}

We thank Prof. Lijun Lin for the help in sample collection. We also thank Wenquan Liang and Zhugui Chen for providing technical support.

\section{References}

1. Martel-Pelletier J, Barr AJ et al. Osteoarthritis. Nat Rev Dis Primers 2016;2:16072

2. Kolasinski SL, Neogi T et al. 2019 American College of Rheumatology/Arthritis Foundation Guideline for the Management of Osteoarthritis of the Hand, Hip, and Knee. Arthritis \& rheumatology (Hoboken, N.J.) 2020;72:220-33

3. Hunter DJ and Bierma-Zeinstra S. Osteoarthritis. Lancet 2019;393:1745-59

4. Cao P, Li Y et al. Pharmacotherapy for knee osteoarthritis: current and emerging therapies. Expert Opin Pharmacother 2020;1-13

5. Clockaerts S, Bastiaansen-Jenniskens YM et al. Cytokine production by infrapatellar fat pad can be stimulated by interleukin 1 beta and inhibited by peroxisome proliferator activated receptor alpha agonist. Ann Rheum Dis 2012;71:1012-8

6. Ushiyama T, Chano $T$ et al. Cytokine production in the infrapatellar fat pad: another source of cytokines in knee synovial fluids. Ann Rheum Dis 2003;62:108-12

7. Klein-Wieringa IR, Kloppenburg $M$ et al. The infrapatellar fat pad of patients with osteoarthritis has an inflammatory phenotype. Ann Rheum Dis 2011;70:851-7

8. Conde $J$, Scotece $M$ et al. Differential expression of adipokines in infrapatellar fat pad (IPFP) and synovium of osteoarthritis patients and healthy individuals. Ann Rheum Dis 2014;73:631-3

9. Eymard F, Pigenet A et al. Knee and hip intra-articular adipose tissues (IAATs) compared with autologous subcutaneous adipose tissue: a specific phenotype for a central player in osteoarthritis. Ann Rheum Dis 2017;76:1142-8

10. Bastiaansen-Jenniskens YM, Clockaerts $S$ et al. Infrapatellar fat pad of patients with end-stage osteoarthritis inhibits catabolic mediators in cartilage. Ann Rheum Dis 2012;71:288-94

11. Nishimuta JF, Bendernagel MF et al. Co-culture with infrapatellar fat pad differentially stimulates proteoglycan synthesis and accumulation in cartilage and meniscus tissues. Connect Tissue Res 2017;58:447-55 
12. He J, Jiang $Y$ et al. Infrapatellar fat pad aggravates degeneration of acute traumatized cartilage: a possible role for interleukin-6. Osteoarthritis Cartilage 2017;25:138-45

13. Pan F, Han W et al. A longitudinal study of the association between infrapatellar fat pad maximal area and changes in knee symptoms and structure in older adults. Ann Rheum Dis 2015;74:1818-24

14. Han W, Cai S et al. Infrapatellar fat pad in the knee: is local fat good or bad for knee osteoarthritis? Arthritis Res Ther 2014;16:R145

15. Teichtahl AJ, Wulidasari E et al. A large infrapatellar fat pad protects against knee pain and lateral tibial cartilage volume loss. Arthritis Res Ther 2015;17:318

16. Cai J, Xu J et al. Association Between Infrapatellar Fat Pad Volume and Knee Structural Changes in Patients with Knee Osteoarthritis. J Rheumatol 2015;42:1878-84

17. Han W, Aitken D et al. Association Between Quantitatively Measured Infrapatellar Fat Pad High Signal-Intensity Alteration and Magnetic Resonance Imaging-Assessed Progression of Knee Osteoarthritis. Arthritis Care Res (Hoboken) 2019;71:638-46

18. Wang K, Ding $C$ et al. Signal intensity alteration within infrapatellar fat pad predicts knee replacement within 5 years: data from the Osteoarthritis Initiative. Osteoarthritis Cartilage 2018;26:1345-50

19. Han W, Aitken $D$ et al. Signal intensity alteration in the infrapatellar fat pad at baseline for the prediction of knee symptoms and structure in older adults: a cohort study. Ann Rheum Dis 2016;75:1783-8

20. Han W, Aitken D et al. Hypointense signals in the infrapatellar fat pad assessed by magnetic resonance imaging are associated with knee symptoms and structure in older adults: a cohort study. Arthritis Res Ther 2016;18:234

21. Krasnokutsky S, Attur M et al. Current concepts in the pathogenesis of osteoarthritis. Osteoarthritis Cartilage 2008;16 Suppl 3:S1-3

22. Kapoor $\mathrm{M}$, Martel-Pelletier $\mathrm{J}$ et al. Role of proinflammatory cytokines in the pathophysiology of osteoarthritis. Nat Rev Rheumatol 2011;7:33-42

23. Gross JB, Guillaume $C$ et al. The infrapatellar fat pad induces inflammatory and degradative effects in articular cells but not through leptin or adiponectin. Clin Exp Rheumatol 2017;35:53-60

24. Rigoglou $S$ and Papavassiliou AG. The NF-kappaB signalling pathway in osteoarthritis. Int $J$ Biochem Cell Biol 2013;45:2580-4

25. Pal B, Endisha H et al. mTOR: a potential therapeutic target in osteoarthritis? Drugs R D 2015;15:2736

26. Sondergaard BC, Schultz $\mathrm{N}$ et al. MAPKs are essential upstream signaling pathways in proteolytic cartilage degradation--divergence in pathways leading to aggrecanase and MMP-mediated articular cartilage degradation. Osteoarthritis Cartilage 2010;18:279-88

27. Jiang LF, Fang JH et al. Role of infrapatellar fat pad in pathological process of knee osteoarthritis: Future applications in treatment. World J Clin Cases 2019;7:2134-42 
28. Chen WH, Lin CM et al. Functional Recovery in Osteoarthritic Chondrocytes Through Hyaluronic Acid and Platelet-Rich Plasma-Inhibited Infrapatellar Fat Pad Adipocytes. Am J Sports Med 2016;44:2696705

29. Hui W, Litherland GJ et al. Leptin produced by joint white adipose tissue induces cartilage degradation via upregulation and activation of matrix metalloproteinases. Ann Rheum Dis 2012;71:455-62

30. Schafer S, Viswanathan S et al. IL-11 is a crucial determinant of cardiovascular fibrosis. Nature 2017; $552: 110-5$

31. Badger AM, Roshak AK et al. Differential effects of SB 242235, a selective p38 mitogen-activated protein kinase inhibitor, on IL-1 treated bovine and human cartilage/chondrocyte cultures. Osteoarthritis Cartilage 2000;8:434-43

32. Chang J, Liao Z et al. Systemic and local adipose tissue in knee osteoarthritis. Osteoarthritis Cartilage 2018;26:864-71

33. Fontanella CG, Macchi V et al. Biomechanical behavior of Hoffa's fat pad in healthy and osteoarthritic conditions: histological and mechanical investigations. Australas Phys Eng Sci Med 2018;41:657-67

34. Zhang $\mathrm{H}$, Wang $\mathrm{H}$ et al. mTORC1 activation downregulates FGFR3 and PTH/PTHrP receptor in articular chondrocytes to initiate osteoarthritis. Osteoarthritis Cartilage 2017;25:952-63

35. Wang $P, M a o ~ Z$ et al. Histone deacetylase -4 and histone deacetylase- 8 regulate interleukin- 1 betainduced cartilage catabolic degradation through MAPK/JNK and ERK pathways. Int J Mol Med 2018;41:2117-27

36. Ge HX, Zou FM et al. JNK pathway in osteoarthritis: pathological and therapeutic aspects. J Recept Signal Transduct Res 2017;37:431-6

37. Fan Z, Bau B et al. IL-1 beta induction of IL-6 and LIF in normal articular human chondrocytes involves the ERK, p38 and NFkappaB signaling pathways. Cytokine 2004;28:17-24

\section{Figures}


A

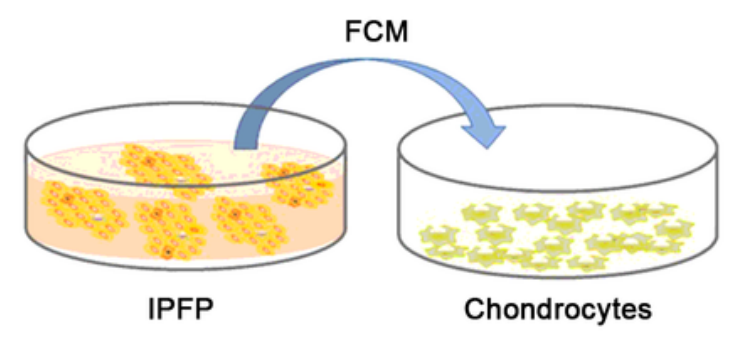

C

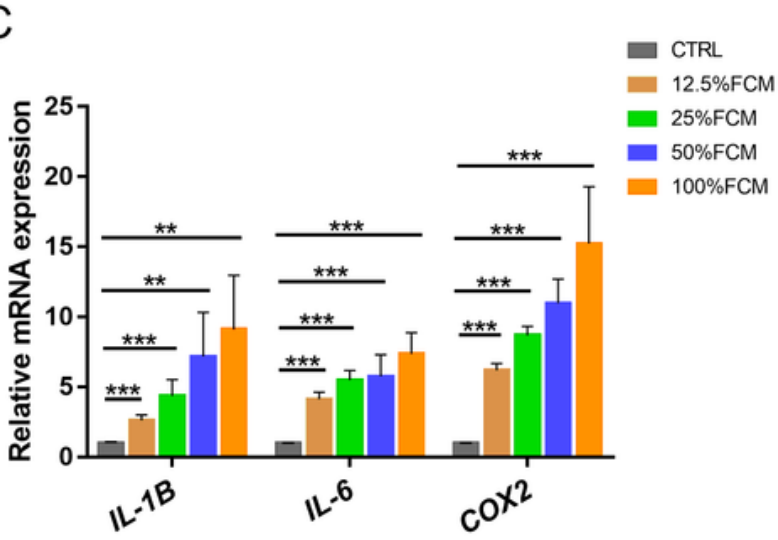

E

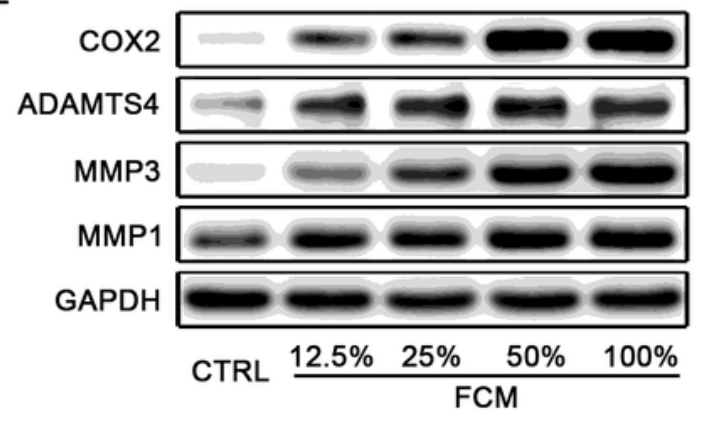

B

$\mathrm{D}$
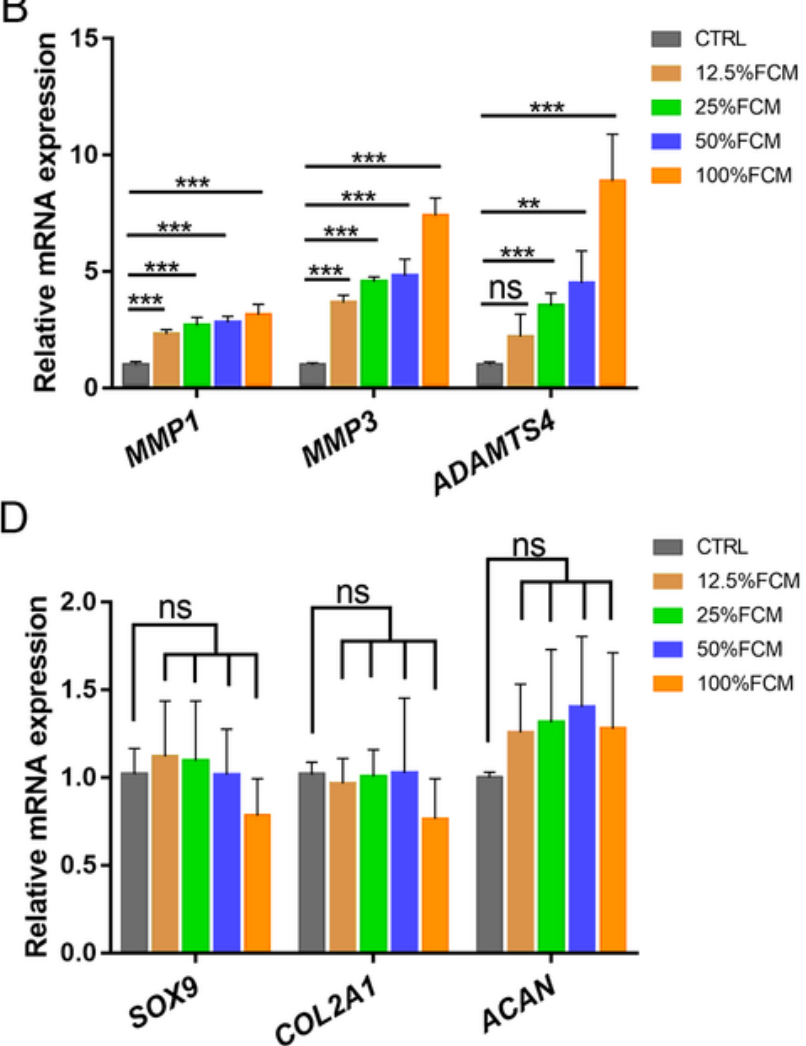

$\mathrm{F}$

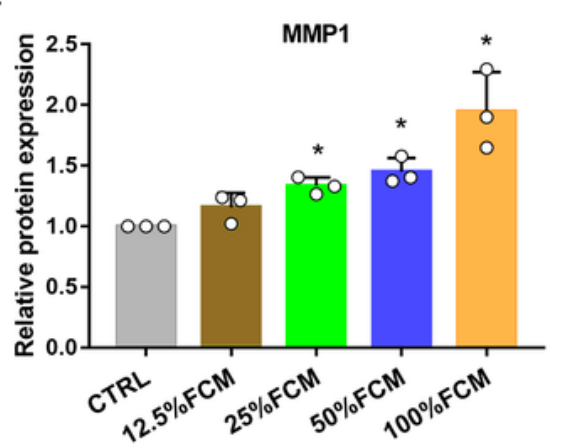

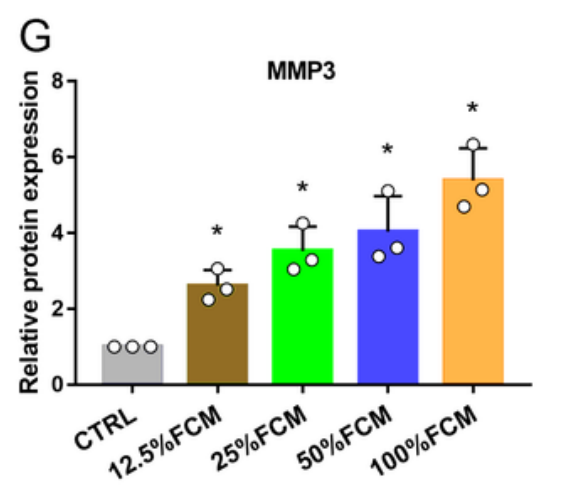

$\mathrm{H}$
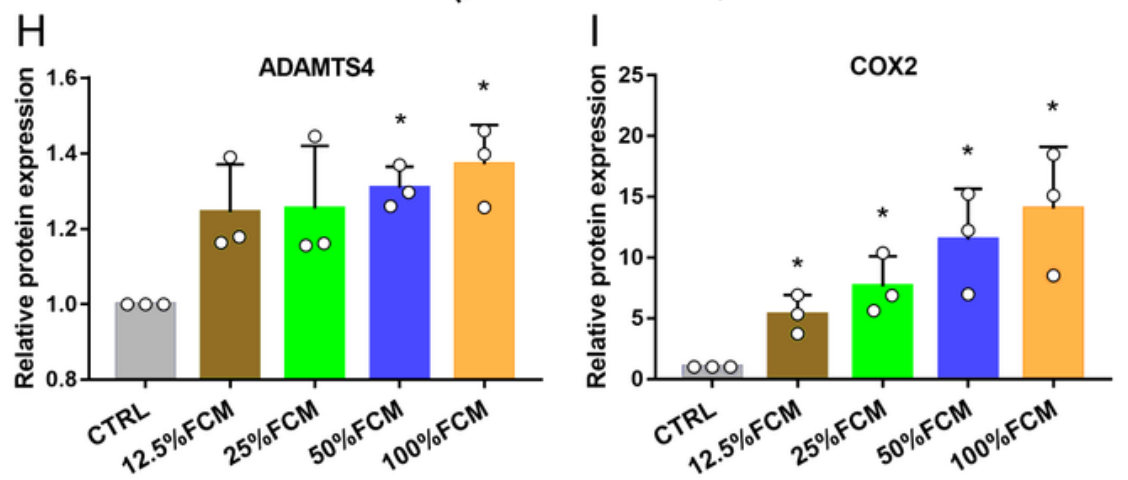

\section{Figure 1}

The effects of FCM from osteoarthritic IPFP on articular chondrocytes. (A) Schematic illustration showing the IPFP-derived FCM stimulus on chondrocytes. (B-D) Gene expressions of (B) degrading enzymes (MMP1, MMP3 and ADAMTS4), (C) inflammatory mediators (IL-1B, IL-6, and COX2) and (D) chondrogenic-specific markers (SOX9, COL2A1 and ACAN) were evaluated by qRT-PCR after $24 \mathrm{~h}$ of treatment with different percentages of FCM and compared with a relative control group (CTRL) cultured 
without FCM $(n=8)$. (E) Western blotting and $(F-1)$ densitometry analysis of MMP1, MMP3, ADAMTS4 and COX2 proteins from cultured chondrocytes treated with different percentages of FCM for $24 \mathrm{~h}(\mathrm{n}=3)$.

Representative results are shown as mean $\pm S D$, Statistical difference: $n s$, not significant, ${ }^{*} P<0.05,{ }^{*} P<$ 0.01 and ${ }^{* \star *} \mathrm{P}<0.001$ compared with the CTRL. FCM, fat conditioned medium; IPFP, infrapatellar fat pad; MMP, matrix metalloproteinase; ADAMTS, A disintegrin and metalloproteinase with thrombospondin-like motifs; IL, interleukin; COX2, cyclooxygenase 2; ACAN, aggrecan; GAPDH, glyceraldehyde-phosphate dehydrogenase

A
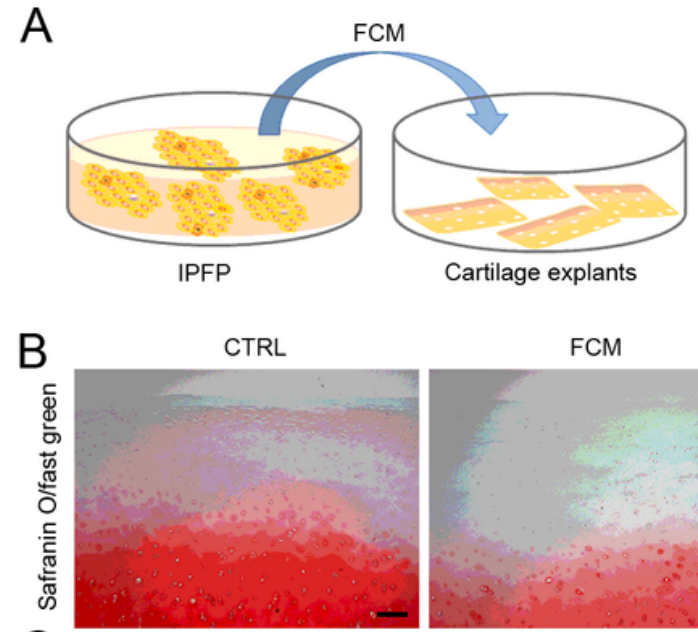

C

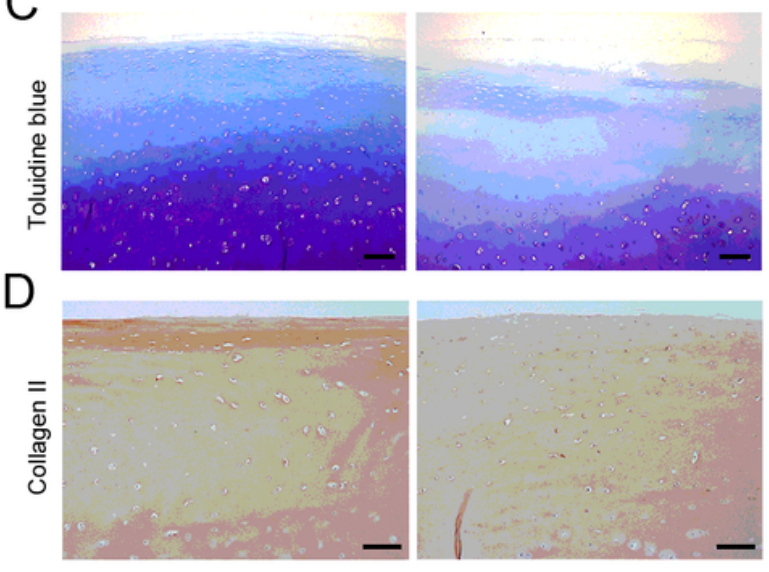

E

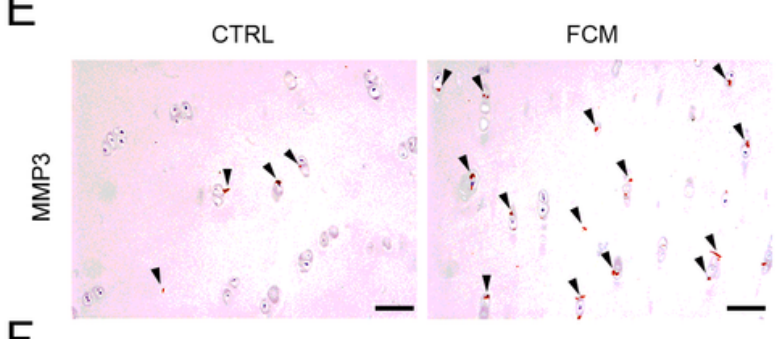

$\mathrm{F}$

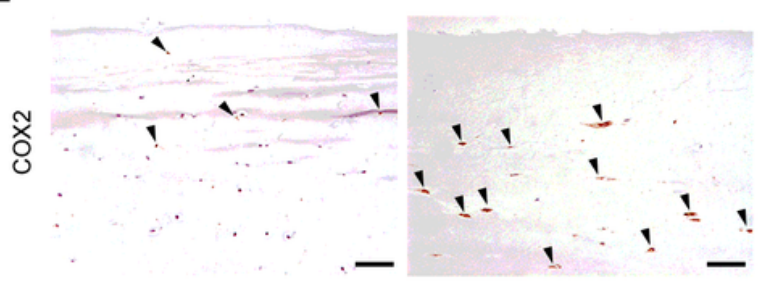

G

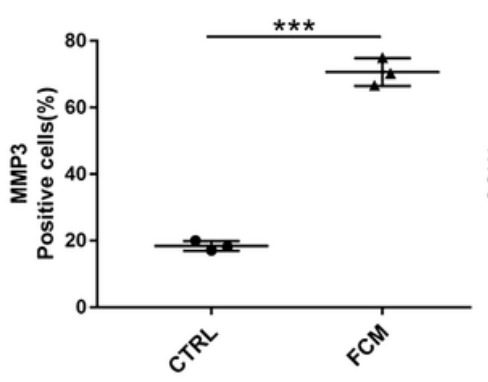

$\mathrm{H}$

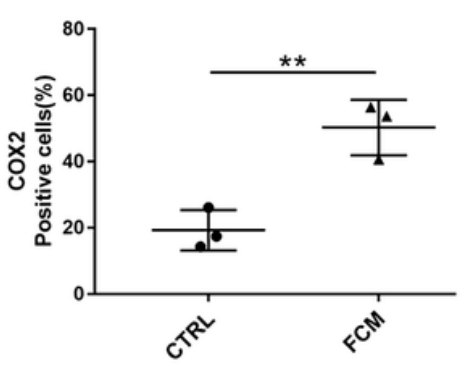

\section{Figure 2}

Osteoarthritic IPFP-derived FCM induced degradative and inflammatory effects on cartilage explants ex vivo. (A) Schematic illustration showing the IPFP-derived FCM stimulus on cartilage. Representative safranin $0 /$ fast green-stained images (B) and toluidine blue-stained images (C) of human cartilage explants treated with or without FCM for 14 days. (D-H) Representative images of immunohistochemistry of Collagen II (D), MMP3 (E) and COX2 (F), and quantification of relative MMP3 (G) or COX2 (H) positive cells as a proportion of total chondrocytes in cartilage explants. $N=3$. Data expressed as mean $\pm S D$. Student's t-test. ${ }^{*} P<0.05,{ }^{*} \mathrm{P}<0.01$. ${ }^{* *} \mathrm{P}<0.001$. FCM, fat conditioned medium; IPFP, infrapatellar fat pad 
A
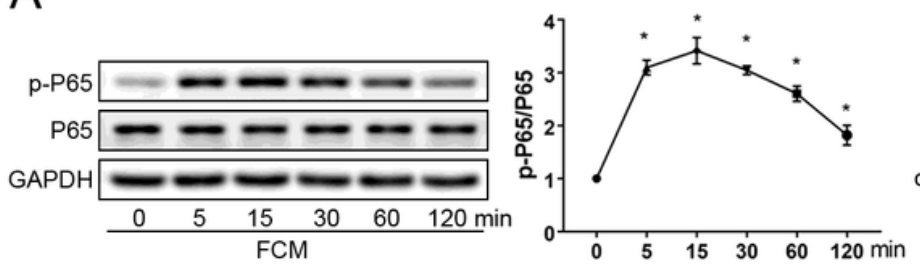

C p-P38 $=-\cdots$

$\mathrm{P} 38 \longrightarrow-\div$
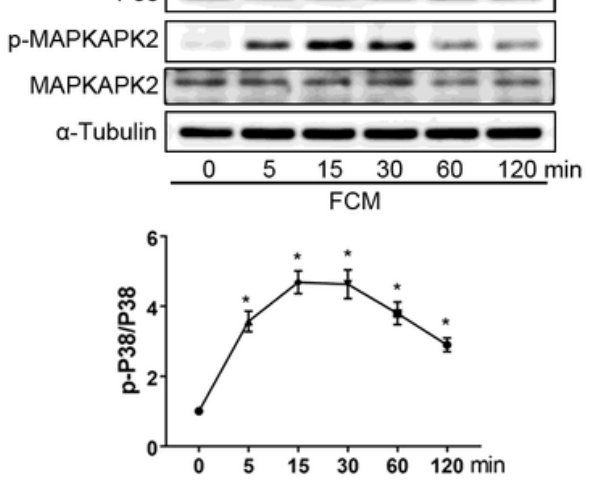

$\mathrm{B}$
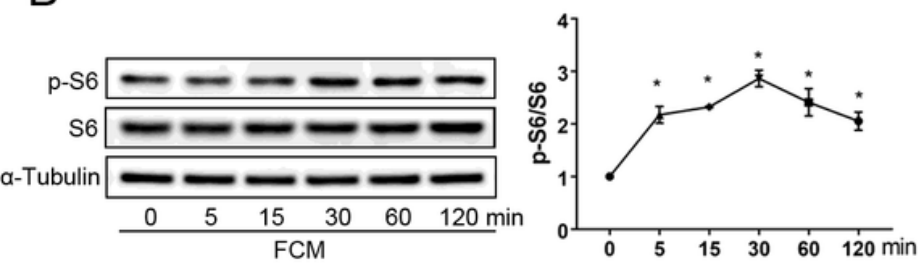

E
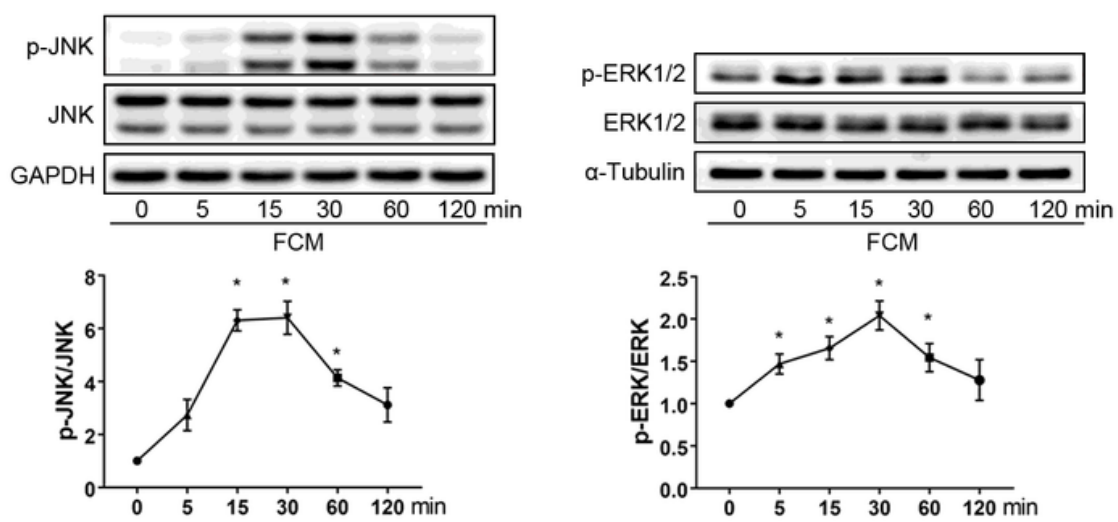

\section{Figure 3}

NF-KB, mTORC1, p38MAPK, JNK, and ERK1/2 signaling pathways were activated in human chondrocytes treated with $50 \%$ FCM from the patients with knee OA. Chondrocytes were incubated for the indicated time points with 50\% FCM, then, P65 (A), S6 (B), P38/MAPKAPK2 (C), JNK (D), and ERK1/2 (E) activations were assessed by western blotting and densitometry analysis of total and phosphorylated protein bands $(n=3)$. GAPDH or a-Tubulin was used as a housekeeping protein. ${ }^{*} P<0.05$ versus control group (time point of $0 \mathrm{~min}$ ). MAPKAPK2, a downstream protein of p38MAPK signaling 
A
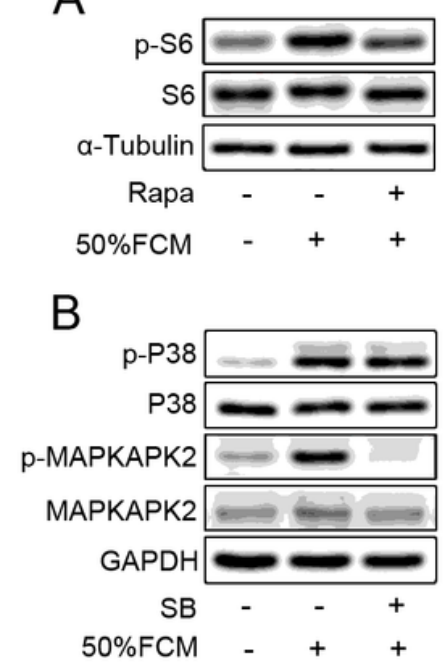

C

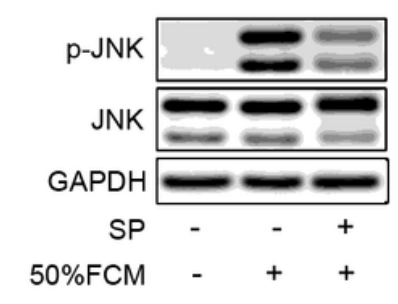

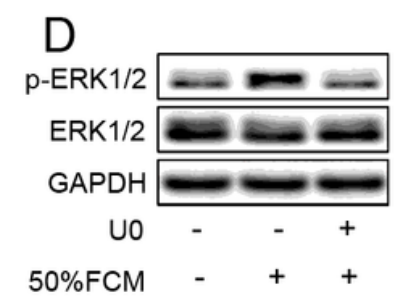

$E$

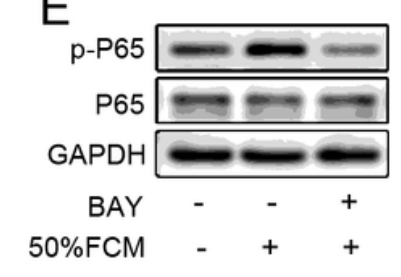

G

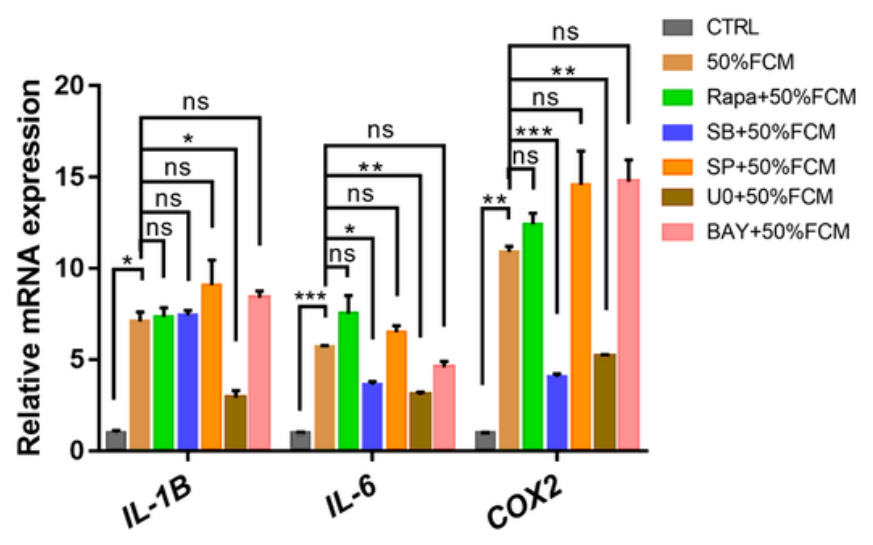

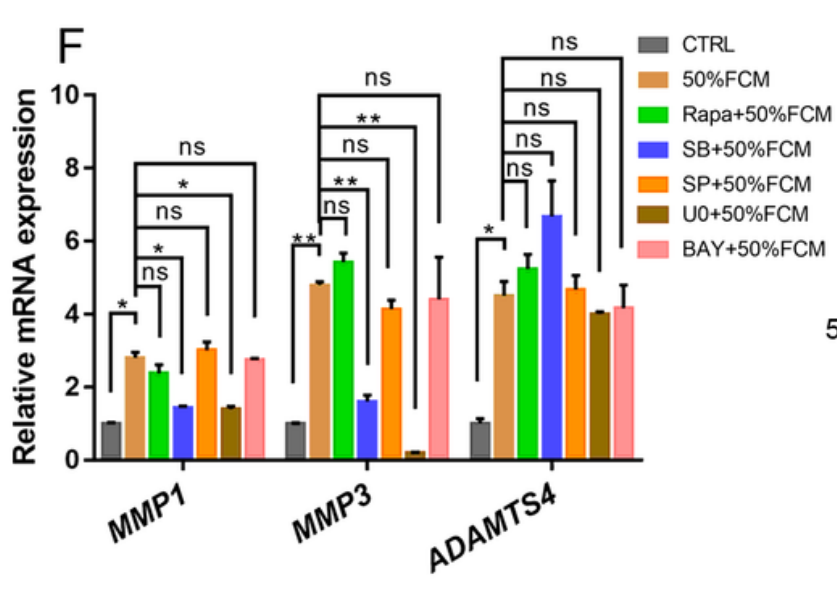

\section{Figure 4}

Inflammatory and degradative responses of chondrocytes to FCM decreased when p38MAPK and ERK1/2 pathways, but not the mTORC1, NF-KB, and JNK pathways, were inhibited. (A-E) Western blotting verified the pathway inhibition effects of relative inhibitors including rapamycin (Rapa, $10 \mathrm{nmol} / \mathrm{l}$, an mTORC1 inhibitor), SB203580 (SB, $10 \mu \mathrm{mol} / \mathrm{l}$, a p38MAPK inhibitor), SP600125 (SP, 5 umol/l, a JNK inhibitor), U0126 (U0, $10 \mu \mathrm{mol} / \mathrm{l}$, an ERK1/2 inhibitor) and BAY11-7082 (BAY, $1 \mu \mathrm{mol} / \mathrm{l}$, an NF-KB inhibitor). Chondrocytes were preincubated with BAY, Rapa, SB, SP, or U0 for $2 \mathrm{~h}$ before treatment with $50 \%$ FCM for 24 h. qRT-PCR analysis of (F) degrading enzymes (MMP1, MMP3, and ADAMTS4) and (G) inflammatory mediators (IL-1B, IL-6, and COX2) expression. (H) Western blotting analysis of MMP1, MMP3, and COX2 expression in indicated groups. $\mathrm{N}=3$. Results were shown as mean $\pm S D$, Statistical difference: $\mathrm{ns}$, not significant, ${ }^{*} P<0.05,{ }^{*} \mathrm{P}<0.01$ and ${ }^{\star * \star P} \mathrm{P}<0.001$ compared with the $\mathrm{CTRL}$ or $50 \%$ FCM group. FCM, fat conditioned medium; CTRL, control group 
A

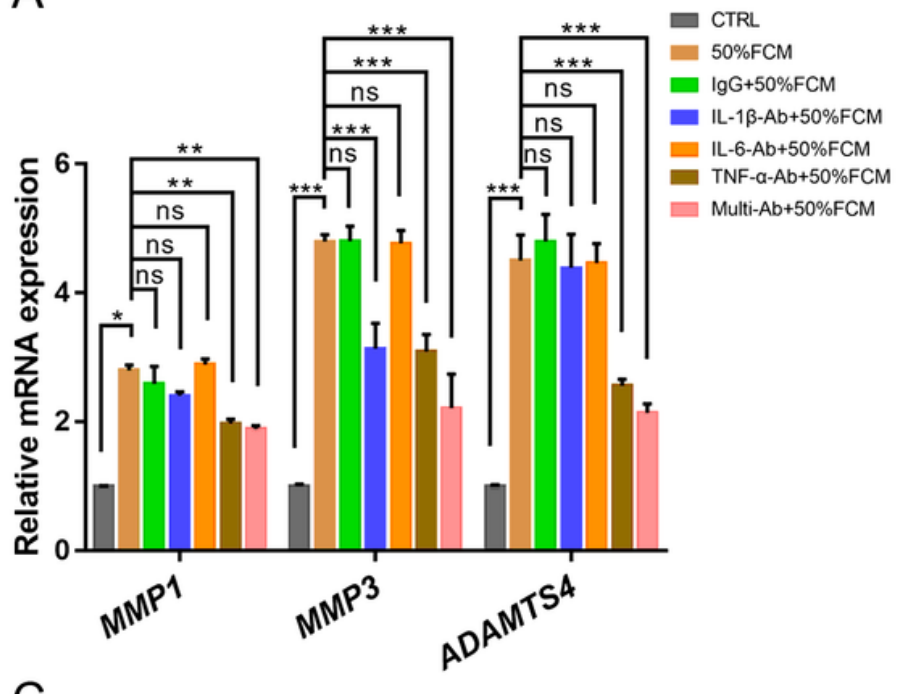

C

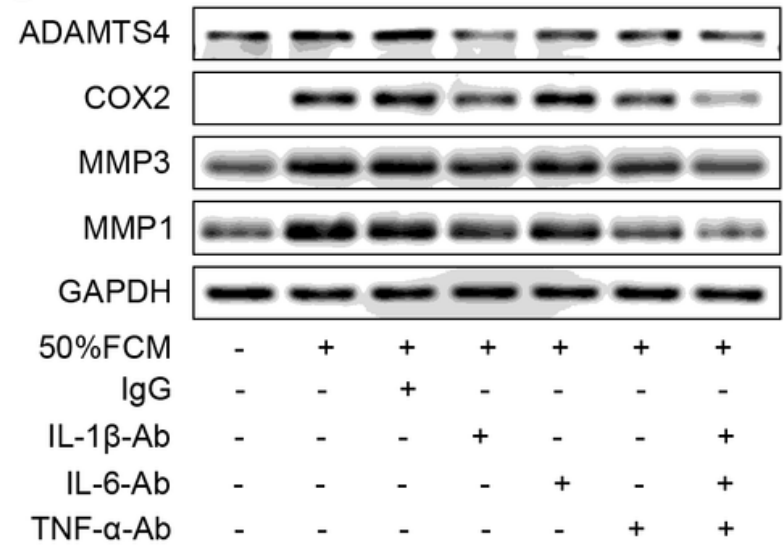

$E$

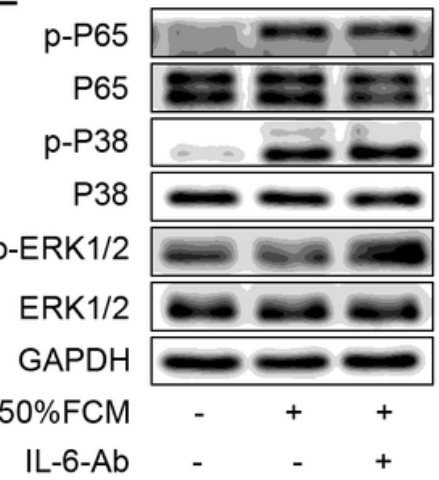

B

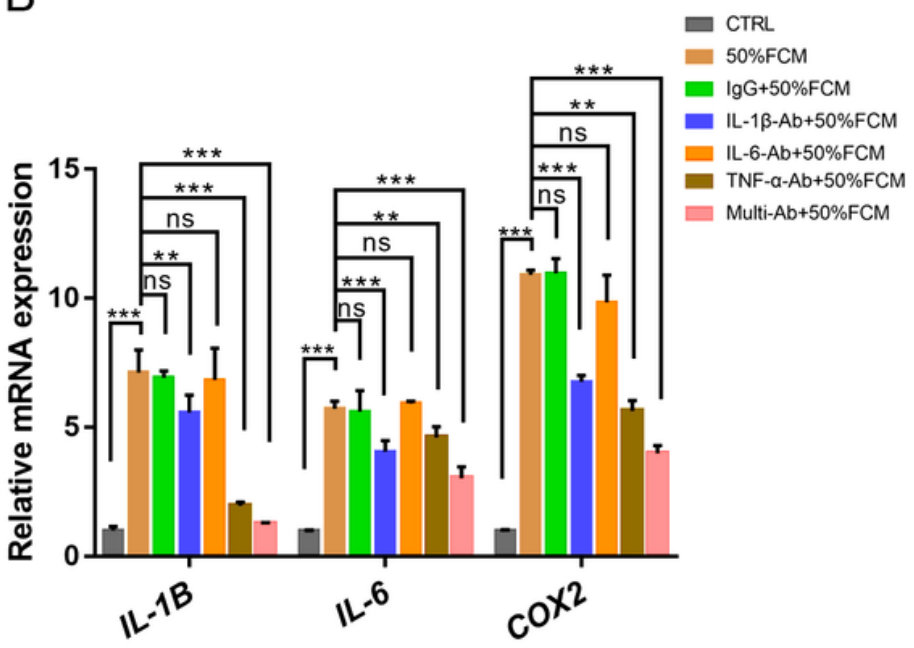

D

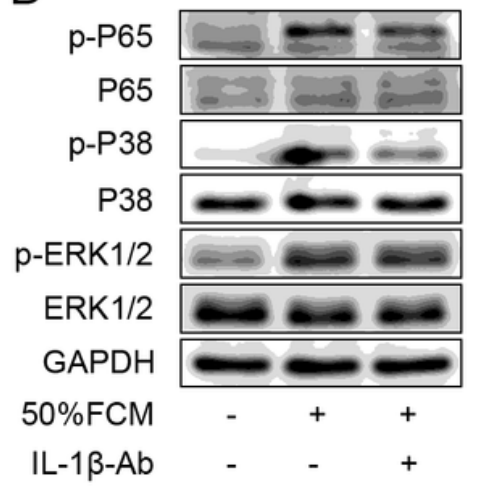

\section{Figure 5}

Pro-inflammatory and degrading effects of FCM on chondrocytes diminished after neutralization of relative cytokines (IL-1 $\beta$ and TNF- $\alpha$ ) in FCM due to the inhibition of p38MAPK signaling. FCM was pretreated with mouse normal IgG, mouse anti-human IL-1 $\beta$, IL-6, TNF- $\alpha$ antibody or multiple antibodies targeting IL-1 $\beta$, IL-6 and TNF- $\alpha$ overnight, then incubated with human chondrocytes for $24 \mathrm{~h}$. Blank control group did not use FCM. qRT-PCR analysis of (A) degrading enzymes (MMP1, MMP3, and ADAMTS4) and (B) inflammatory mediators (IL-1B, IL-6, and COX2) expression $(n=3)$. (C) Western blotting analysis of 
the protein levels of MMP1, MMP3, COX2 and ADAMTS4 in different groups. (D-G) Western blotting analysis of the effects of blocking IL-1 $\beta(D)$, IL-6 (E), TNF-a (F), or all of them (G) in FCM on pathway activation including NF-KB, p38MAPK and ERK1/2 signaling. Results expressed as mean \pm SD. Statistical difference: ns, not significant, $* \mathrm{P}<0.05$, $* * \mathrm{P}<0.01$ and $* \star * P<0.001$ compared with the CTRL or $50 \% \mathrm{FCM}$ group. CTRL, control group; FCM, fat conditioned medium; IL-1 $\beta-A b$, IL-6-Ab, TNF- $a-A b$ and Multi-Ab represented neutralizing antibodies targeting IL-1 $\beta$, IL-6, TNF- $\alpha$ and all of them, respectively.

\section{Osteoarthritic infrapatellar fat pad}

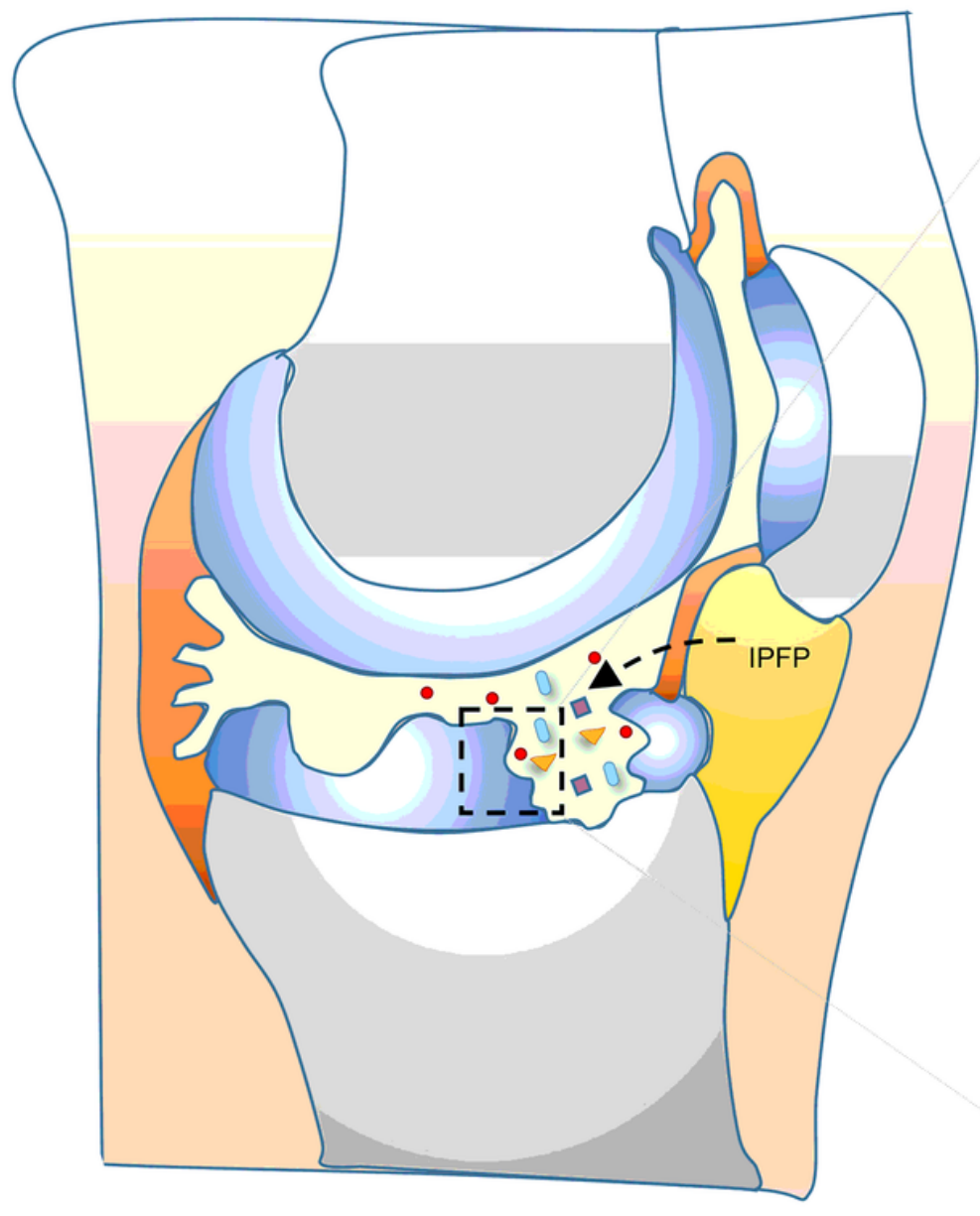

IL-1 $\beta \quad$ TNF $\alpha \quad$ Other cytokines

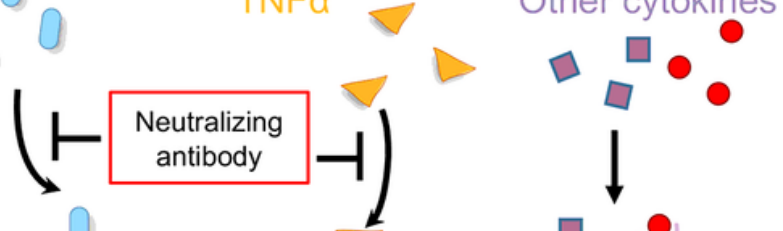

if
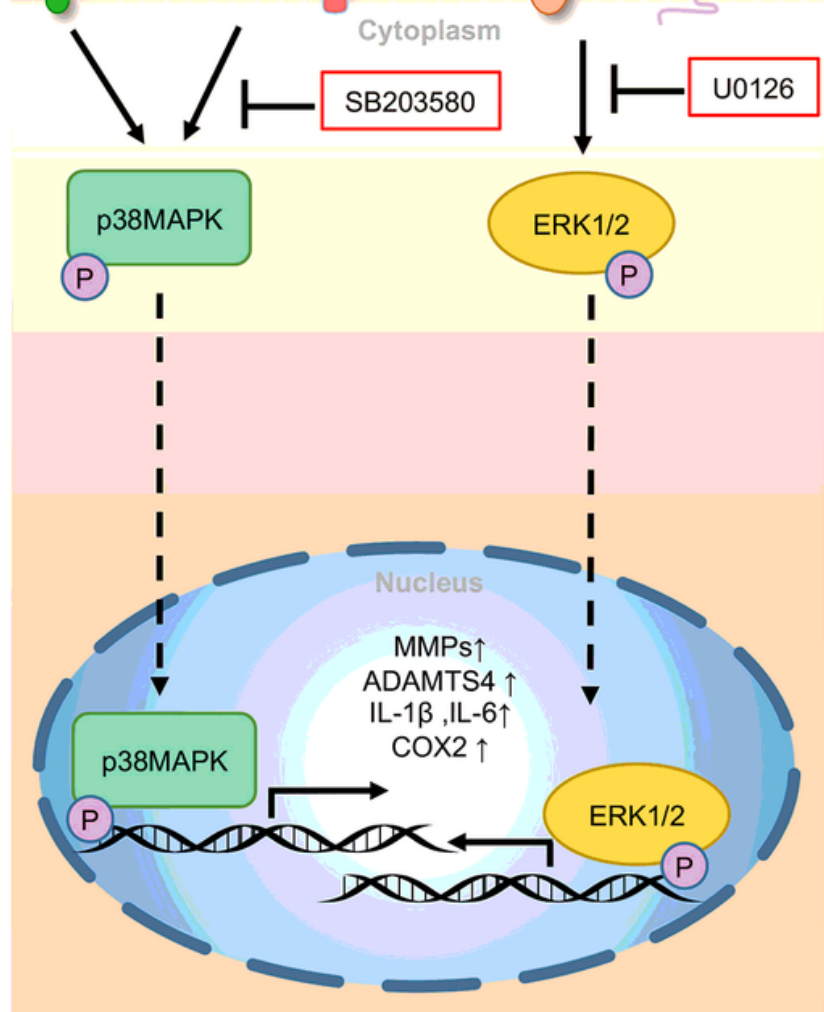

Figure 6

Schematic working model showing the biochemical effects of osteoarthritic IPFP on cartilage. Osteoarthritic IPFP released factors activate different molecular pathways, especially the p38MAPK and ERK1/2 signaling, in which, IL-1 $\beta$ and TNF-a rather than IL- 6 are the key players. IPFP, infrapatellar fat pad; IL, interleukin; TNF-a, tumor necrosis factor-a; MAPK, mitogen-activated protein kinase; ERK, extracellular signal-regulated kinase. SB203580, a p38MAPK inhibitor; U0126, an ERK1/2 inhibitor; MMP, matrix metalloproteinase; ADAMTS, A disintegrin and metalloproteinase with thrombospondin-like motifs; COX2, cyclooxygenase 2 . 


\section{Supplementary Files}

This is a list of supplementary files associated with this preprint. Click to download.

- SupplementaryFigureS1.tif

- Supplement20201022docx.docx 